\title{
Migraine and Ischemic Stroke: A Mendelian Randomization Study
}

\author{
Mei-Jun Shu $\cdot$ Jia-Rui Li $\cdot$ Yi-Cheng Zhu $\cdot$ Hang Shen
}

Received: September 23, 2021 / Accepted: November 26, 2021 / Published online: December 13, 2021

(c) The Author(s) 2021

\begin{abstract}
Introduction: Previous epidemiological studies have found an increased risk for ischemic stroke in patients with migraine; however, the evidence for a causal relationship between migraine and ischemic stroke is scarce. This study aims to explore the potential causal relationship between migraine and ischemic stroke and its subtypes [including large artery stroke (LAS), small vessel stroke (SVS), and cardioembolic stroke (CES)].

Methods: We used data on genetic variants associated with migraine identified from a genome-wide association study (GWAS) metaanalysis among 889,018 European ancestries. Summary data for ischemic stroke and its subtypes were obtained from the MEGASTROKE
\end{abstract}

Supplementary Information The online version contains supplementary material available at https:// doi.org/10.1007/s40120-021-00310-y.

M.-J. Shu · Y.-C. Zhu · H. Shen ( $₫)$

Department of Neurology, Peking Union Medical College Hospital, Chinese Academy of Medical Sciences and Peking Union Medical College, No. 1 Shuaifuyuan, Wangfujing, Beijing 10073, China e-mail: shenhang12@sina.com

J.-R. Li

Department of Oncology of Peking Union Medical College Hospital, Chinese Academy of Medical Sciences and Peking Union Medical College, Beijing, China consortium including up to 438,847 participants. We performed two-sample Mendelian randomization (MR) analyses using the inversevariance-weighted method as the primary approach. The MR-Egger, weighted median, simple median, simple mode, and weighted mode methods were also conducted as sensitivity analyses to determine the robustness of our results.

Results: We failed to detect statistically significant associations between migraine and ischemic stroke (OR, 0.935; 95\% CI $0.851-1.027 ; P=0.159)$ and its subtypes (LAS: OR, 0.818; 95\% CI 0.692-0.967; $P=0.018$ ) (SVS: OR, $0.935 ; 95 \%$ CI $0.781-1.119 ; \quad P=0.460$ ) (CES: OR, 1.015; 95\% CI $0.867-1.189$; $P=0.850)$. The results were consistent with the sensitivity analyses.

Conclusions: By conducting a series of causal inference approaches, this study supports no causal effect of migraine on ischemic stroke and its subtypes.

Keywords: Migraine; Stroke; Ischemic stroke; Mendelian randomization 


\section{Key Summary Points}

\section{Why carry out this study?}

Although some studies have failed to find any association, there is growing evidence of a possible association between migraine and ischemic stroke. It is still unknown, however, whether these associations are causal or confounded.

In this study, we aimed to investigate the causal relationship between migraine and ischemic stroke using the Mendelian randomization approach.

\section{What was learned from the study?}

In this two-sample Mendelian randomization analysis, we did not find causality between migraine and ischemic stroke and its subtypes.

This study suggests that migraine-specific pharmacological interventions are not required for the primary prevention of ischemic stroke in patients with migraine.

\section{INTRODUCTION}

Stroke and migraine, which rank as the most common neurological disorders, are a principal cause of death and disability worldwide, with a high socioeconomic burden [1]. Previous epidemiological studies have observed an increased risk for ischemic stroke in patients with migraine [2]. However, the strength and significance of the observed migraine-stroke association are still up for debate [3]. In a Swedish population-based twin cohort [4], there was no evidence for the existence of an association between stroke risk and migraine overall, though an increased stroke risk related to migraine with aura was found.

The underlying mechanisms linking migraine to stroke events remain inconclusive, though several hypotheses, including cortical spreading depression theory, have been proposed to explain the pathogenic mechanisms of the migraine-stroke association [5,6]. In observational epidemiological studies, however, causality is rarely proven, even if there is a strong statistical correlation [7]. A majority of the previous studies concerning the relationship between migraine and stroke have been observational, leaving the relationship to be considered tenuous, with reverse causality and existing confounding factors unable to be excluded. The most recent and largest genomewide association studies (GWAS) of migraine [8] and stroke [9] provide a new perspective and way to help determine whether migraine is an independent risk factor for stroke occurrence. The Mendelian randomization (MR) method, which employs genetic variants as instrumental variables (IVs) to infer the causality of an association, effectively overcomes bias due to confounding and reverse causality issues in observational epidemiological studies [10].

Notably, current guidelines do not recommend the use of antithrombotic drugs in migraine prophylaxis $[11,12]$. If causality between migraine and stroke exists, however, the benefit of preventive medicine in migraine patients needs to be further verified in randomized controlled trials (RCTs) and cost-effectiveness analysis. In this study, we aimed to investigate the causal relationship between migraine and ischemic stroke using the MR approach.

\section{METHODS}

\section{Mendelian Randomization Assumptions}

The MR method is an instrumental variables analysis that uses genetic variants (e.g., singlenucleotide polymorphisms or SNPs) as proxies for exposure. Three key assumptions [13] need to be satisfied to ensure the selected SNPs as valid IVs: (1) SNPs used as IVs are associated with the exposure (migraine); (2) the genetic variants affect ischemic stroke only via their effects on migraine, not through any other causal pathway; and (3) the genetic variants 


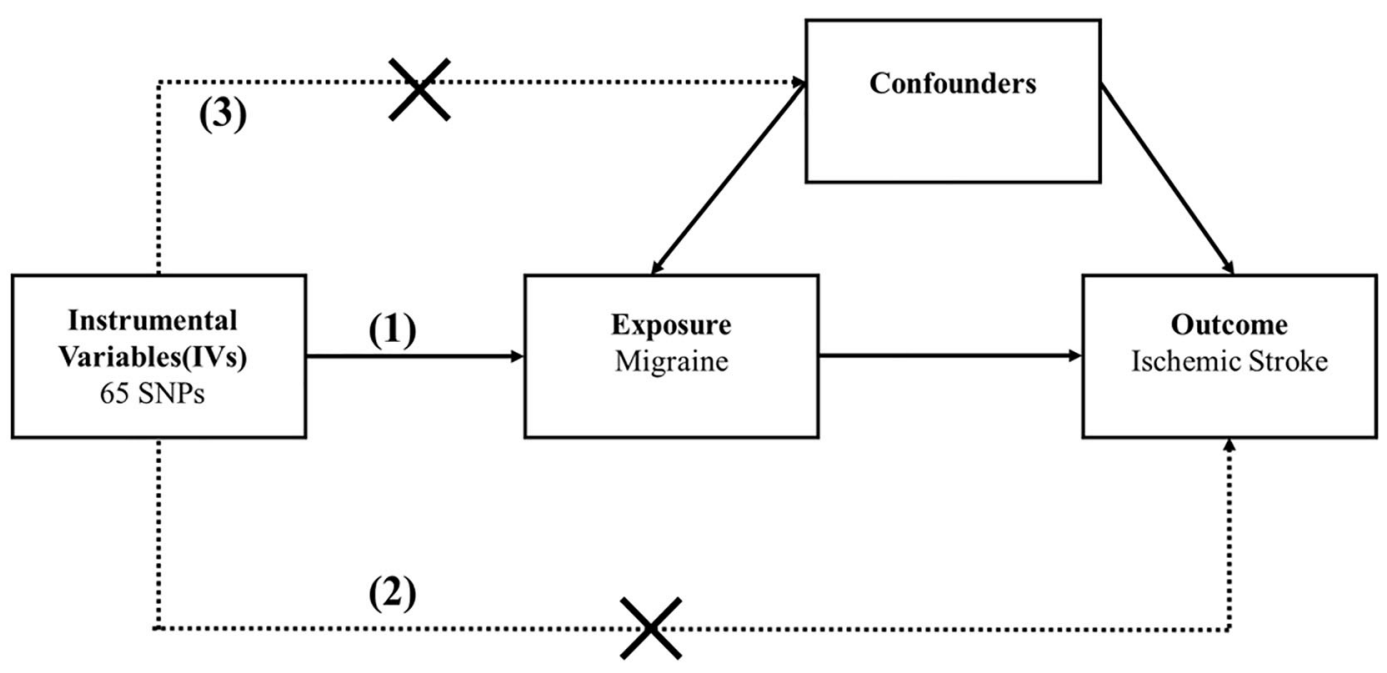

Fig. 1 Design and main assumptions of our Mendelian randomization study. SNPs single-nucleotide polymorphisms

must not be associated with measured or unmeasured confounders (Fig. 1).

\section{Selection of Genetic Variants}

We used data on genetic variants associated with migraine from the largest GWAS metaanalysis, which included 889,018 participants $(85,726$ migraine cases) [8]. This data set consists of the European ancestry from the Genetic Epidemiology Research on Adult Health and Aging (GERA) cohort, the UK Biobank (UKB) cohort, and GWAS summary statistics data from the study by Gormley et al. [14]. For the first key assumption of our MR analysis, we selected genetic variants associated with migraine at a genome-wide significance threshold $\left(P<5 \times 10^{-8}\right)$ as the candidate IVs. In total, 73 SNPs were extracted at a genome-wide significance threshold (Supplementary Table 1); independent SNPs were selected at a threshold of linkage disequilibrium clumping $r^{2}<0.001$ over a 10-kilobase $(\mathrm{kb})$ region based on the European sample of 1000 Genomes data [15] (accounts for SNP correlations). Of all 73 SNPs, eight SNPs were removed due to linkage disequilibrium (rs4704232, rs12936464, rs75002882, rs7093087, rs1268083, rs6693567, rs4278348, rs1026332), leaving 65 SNPs remaining.
Given the high comorbidity associated with stroke and migraine [14], possible confounders must be considered. We applied the PhenoScanner (http://www.phenoscanner.medschl. cam.ac.uk/phenoscanner) to assess whether the selected SNPs were associated with other traits at genome-wide significance levels, which might violate the second and third key assumptions. In the SNPs related to migraine at a genome-wide significance threshold, we identified eight SNPs (rs10456100, rs138556413, rs1800469, rs2000660, rs28451064, rs4888378, rs8075138, and rs9349379) also associated with vascular events, as well as one SNP for body mass index (BMI) (rs8054079) and five SNPs for systolic blood pressure (rs10786156, rs4888378, rs4909945, rs9349379, and rs11153082). We then evaluated the results after excluding these pleiotropic SNPs. The F-statistic of the selected SNPs was calculated to test the weak IV bias for our MR study. The $F$-statistics of the selected IVs were all above the threshold of weak instruments of $F$ statistic $<10$, indicating strong IVs for the MR study [16].

\section{Data Sources for Outcomes}

GWAS summary data on ischemic stroke and its subtypes were obtained from the GWAS metaanalysis of the MEGASTROKE consortium, 
which included 438,847 individuals of European descent (40,585 cases; 406,111 controls) [9]. Ischemic stroke was defined according to the Trial of Org 10172 in Acute Stroke Treatment (TOAST) classification system, and further subtyped as large artery stroke (LAS), cardioembolic stroke (CES), and small vessel stroke (SVS). For more detailed information on sample description, genotyping, and statistical analysis, please refer to the original paper [9].

\section{Statistical Analysis}

We performed two-sample MR analyses using the inverse-variance-weighted method as the primary approach [17]. We also employed several other MR approaches including the MREgger, the inverse-variance-weighted (multiplicative random effects), weighted median, simple median, simple mode, and weighted mode methods to detect the robustness of our results [18]. We further performed a leave-oneout sensitivity analysis to assess whether the results were influenced by individual SNPs.

In addition, several analyses were used to detect heterogeneity and pleiotropy, ensuring that the second and third key assumptions of our MR study were valid. We used the $I^{2}$ index and Cochran's $Q$ statistic for MR-inverse-variance weighted analyses and Rucker's $Q$ statistic for MR-Egger analyses to detect heterogeneity [19]. We used the MR-Egger method to assess the extent to which directional pleiotropy may affect risk estimates by intercept tests. As the MR-Egger might show low accuracy in some circumstances, the MR pleiotropy residual sum and outlier (MR-PRESSO) approach was also used to assess outlier SNPs and potential horizontal pleiotropy [20]. In addition, the MR Steiger directionality test was used to test whether the assumption that exposure causes outcome is valid.

The analyses were conducted using the TwoSampleMR (version 0.5.6) and MR-PRESSO (version 1.0) R packages. All statistical tests were two-tailed. Associations were considered statistically significant at $P$ values below 0.0125 (Bonferroni-corrected for four outcomes).
This article is based on previously conducted studies and does not contain any new studies with human participants or animals performed by any of the authors.

\section{RESULTS}

After linkage disequilibrium clumping and excluding variants that are known to be pleiotropic, we finally identified 53 SNPs as IVs in our MR analyses (Supplementary Table 2). According to the conventional inverse-varianceweighted method, we failed to detect any statistically significant association between migraine and ischemic stroke (OR, 0.935; 95\% CI 0.851-1.027; $P=0.159$ ) (Table 1). Similarly, we did not find any correlation between migraine and LAS (OR, 0.818; 95\% CI $0.692-0.967 ; P=0.018)$, SVS (OR, $0.935 ; 95 \% \mathrm{CI}$ $0.781-1.119 ; P=0.460)$, or CES (OR, $1.015 ; 95 \%$ CI $0.867-1.189 ; P=0.850$ ) (Table 1 ).

To assess the robustness and consistency of the results, we performed a series of sensitivity analyses. In the sensitivity analyses, the MREgger, weighted median, simple median, simple mode, and weighted mode analyses yielded similar estimates of the effect of migraine on ischemic stroke and its subtypes (Table 1). The effects of each instrumental SNP on the risk of ischemic stroke and its subtypes are shown in Supplementary Fig. 1, which shows the consistency and directional effects. Furthermore, the results of the leave-one-out analysis showed that the overall estimates were not driven by individual SNP, but rather an overall combined effect between migraine and ischemic stroke (Fig. 2).

For LAS, the $P$ values of Cochran's $Q$ and Rucker's $Q$ statistic were both $>0.05$ (Cochran's $Q P$ value $=0.329$; Rucker's $Q P$ value $=0.324)$, which suggests no bias due to heterogeneity in this MR analysis (Supplementary Table 3). For ischemic stroke and the SVS and CES subtypes, the $P$ values of Cochran's $Q$ and Rucker's $Q$ statistic were all $<0.050$, which suggests heterogeneity (Supplementary Table 3). However, even when the heterogeneity was taken into consideration using the multiplicative random-effects inverse-variance-weighted 
Table 1 The causal effects of migraine on ischemic stroke and its subtypes using multiple Mendelian randomization methods

\begin{tabular}{|c|c|c|c|c|}
\hline Outcome traits & Mendelian randomization methods & $\begin{array}{l}\text { Number of } \\
\text { SNPs }\end{array}$ & OR $(95 \% \mathrm{CI})$ & $P$ value \\
\hline \multirow{7}{*}{$\begin{array}{l}\text { Any ischemic } \\
\text { stroke }\end{array}$} & Inverse-variance-weighted & 53 & $0.935(0.851,1.027)$ & 0.159 \\
\hline & $\begin{array}{l}\text { Inverse-variance-weighted (multiplicative random } \\
\text { effects) }\end{array}$ & 53 & $0.935(0.851,1.027)$ & 0.159 \\
\hline & MR-Egger & 53 & $0.931(0.726,1.194)$ & 0.575 \\
\hline & Simple median & 53 & $0.908(0.812,1.015)$ & 0.088 \\
\hline & Weighted median & 53 & $0.877(0.789,0.975)$ & 0.015 \\
\hline & Simple mode & 53 & $0.844(0.662,1.074)$ & 0.174 \\
\hline & Weighted mode & 53 & $0.848(0.735,0.979)$ & 0.028 \\
\hline \multirow[t]{7}{*}{ Large artery stroke } & Inverse-variance-weighted & 53 & $0.818(0.692,0.967)$ & 0.018 \\
\hline & $\begin{array}{l}\text { Inverse-variance-weighted (multiplicative random } \\
\text { effects) }\end{array}$ & 53 & $0.818(0.692,0.967)$ & 0.018 \\
\hline & MR-Egger & 53 & $0.676(0.434,1.052)$ & 0.088 \\
\hline & Simple median & 53 & $0.931(0.726,1.195)$ & 0.574 \\
\hline & Weighted median & 53 & $0.752(0.584,0.968)$ & 0.027 \\
\hline & Simple mode & 53 & $1.303(0.715,2.375)$ & 0.392 \\
\hline & Weighted mode & 53 & $0.692(0.467,1.026)$ & 0.072 \\
\hline \multirow[t]{7}{*}{ Small vessel stroke } & Inverse-variance-weighted & 53 & $0.935(0.781,1.119)$ & 0.460 \\
\hline & $\begin{array}{l}\text { Inverse-variance-weighted (multiplicative random } \\
\text { effects) }\end{array}$ & 53 & $0.935(0.781,1.119)$ & 0.460 \\
\hline & MR-Egger & 53 & $1.073(0.665,1.730)$ & 0.775 \\
\hline & Simple median & 53 & $0.924(0.739,1.156)$ & 0.490 \\
\hline & Weighted median & 53 & $0.923(0.730,1.169)$ & 0.507 \\
\hline & Simple mode & 53 & $0.933(0.596,1.462)$ & 0.765 \\
\hline & Weighted mode & 53 & $0.923(0.685,1.244)$ & 0.603 \\
\hline
\end{tabular}


Table 1 continued

\begin{tabular}{lllll}
\hline Outcome traits & Mendelian randomization methods & $\begin{array}{l}\text { Number of } \\
\text { SNPs }\end{array}$ & OR (95\%CI) & $\boldsymbol{P}$ value \\
\hline $\begin{array}{l}\text { Cardioembolic } \\
\text { stroke }\end{array}$ & $\begin{array}{l}\text { Inverse-variance-weighted } \\
\text { Inverse-variance-weighted (multiplicative random } \\
\quad \text { effects) }\end{array}$ & 53 & $1.015(0.867,1.189)$ & 0.850 \\
& MR-Egger & 53 & $1.015(0.867,1.189)$ & 0.850 \\
& Simple median & 53 & $0.981(0.645,1.492)$ & 0.928 \\
& Weighted median & 53 & $0.940(0.766,1.153)$ & 0.550 \\
& Simple mode & 53 & $0.920(0.583,1.451)$ & 0.721 \\
& Weighted mode & 53 & $0.978(0.719,1.329)$ & 0.887 \\
\hline
\end{tabular}

MR-Egger Mendelian randomization-Egger method, $O R$ odds ratio, $C I$ confidence interval, SNP single-nucleotide polymorphism

methods ${ }^{[18]}$, no causal effect was found in the results for ischemic stroke and the SVS and CES subtypes (Table 1). The MR-Egger intercept test showed no evidence of horizontal pleiotropy for the effects of migraine on ischemic stroke (intercept $=2.240 \times 10^{-4} ; P=0.971$ ), LAS (intercept $=0.010 ; \quad P=0.365), \quad$ SVS (intercept $=-0.007 ; P=0.544$ ), or CES (intercept $\left.=1.833 \times 10^{-3} ; \quad P=0.861\right)$. The MRPRESSO test further showed no outlier pleiotropy and indicated no SNP outliers $(P=0.165$ for ischemic stroke; $P=0.297$ for LAS; $P=0.464$ for SVS; $P=0.850$ for CES), together suggesting no evidence of possible pleiotropic effects. The MR Steiger directionality test confirmed that our assumption that exposure (migraine) causes outcome (ischemic stroke and its subtypes) is valid (Supplementary Table 4).

\section{DISCUSSION}

To the best of our knowledge, this is the first large-scale MR study evaluating the causal relationship between migraine and ischemic stroke. This study did not find a causal relationship between migraine and ischemic stroke and its subtypes.
Many epidemiological studies have reported an increased risk of ischemic stroke in patients with migraine [21]. A large meta-analysis of case-control and observational cohort studies reported an increased risk of ischemic stroke in both migraine with aura and migraine without aura [22]. However, epidemiological studies of migraine-associated stroke risk [2] always highlight the consistent connection between migraine with aura and ischemic stroke, while the evidence favoring the relationship between migraine without aura and stroke is insufficient. There is debate about whether aura-specific effects exist. The GWAS data for migraine used in our study did not include analysis for migraine type subclassification, which precludes determination of aura-specific effects. The paper by Gormley et al. in 2016 [14] identified seven specific loci for migraine without aura, but no loci for migraine with aura in the subset GWAS analysis. In their heterogeneity analysis of migraine subtypes [14], the authors further demonstrated that most of the identified migraine susceptibility loci affected risk for both migraine subtypes, indicating the possibility that migraine with aura and migraine without aura might have a shared underlying genetic susceptibility profile. Although we cannot conclude that future larger-scale GWAS will 
A
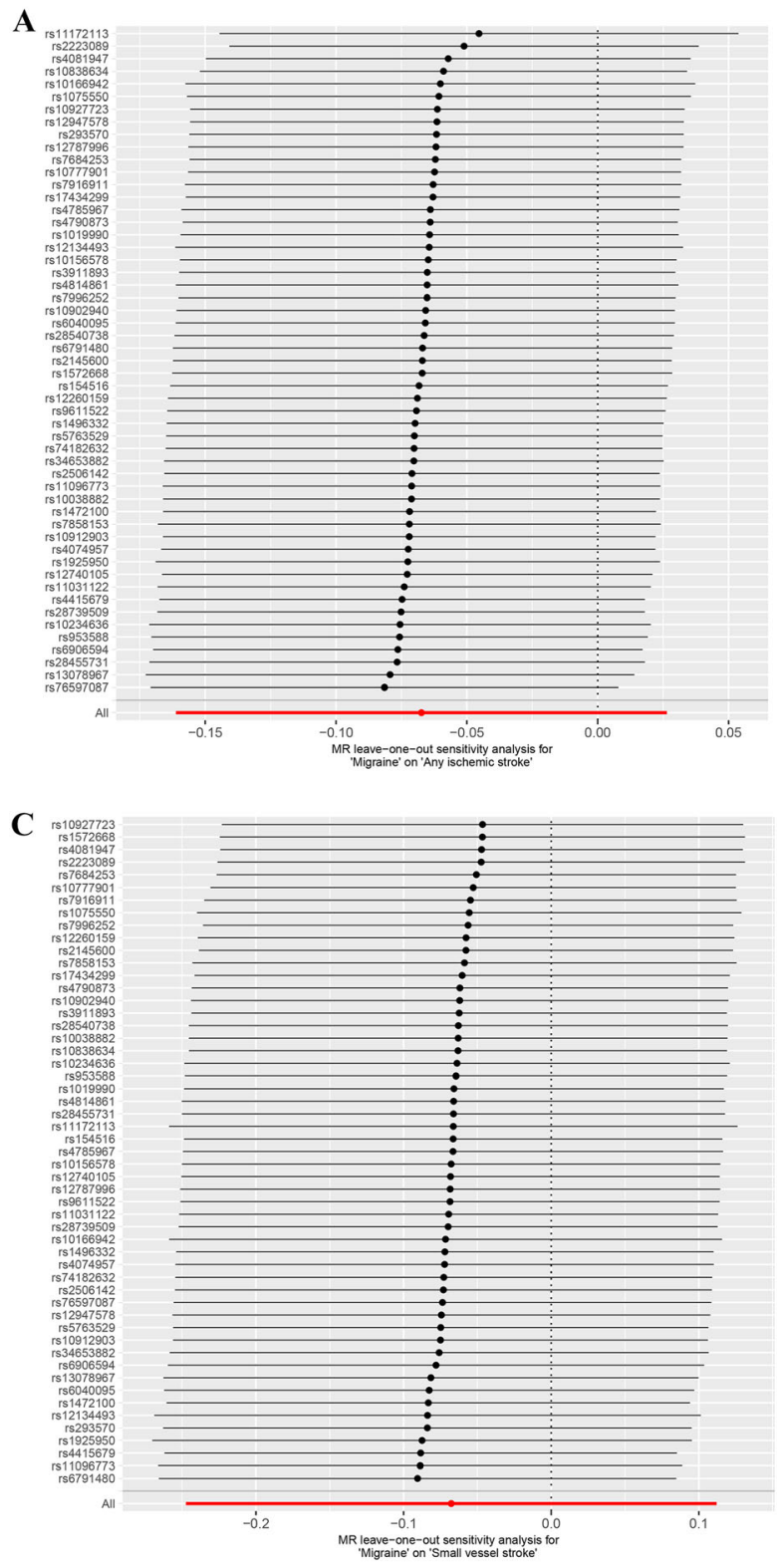

Fig. 2 Leave-one-out analysis of the causal effects with risk for all types and subtypes of ischemic stroke. a All ischemic stroke; b large artery stroke; c small vessel stroke; d cardioembolic stroke. The black dots and bars indicate the causal estimate and 95\% CI when a SNP was removed

never find some specific loci for migraine with aura, we can reasonably assume that migraine with aura and migraine without aura are not distinct entities in our research.

Malik et al. [23] reported a shared genetic basis for migraine and ischemic stroke by applying a polygenic risk score. They concluded
B

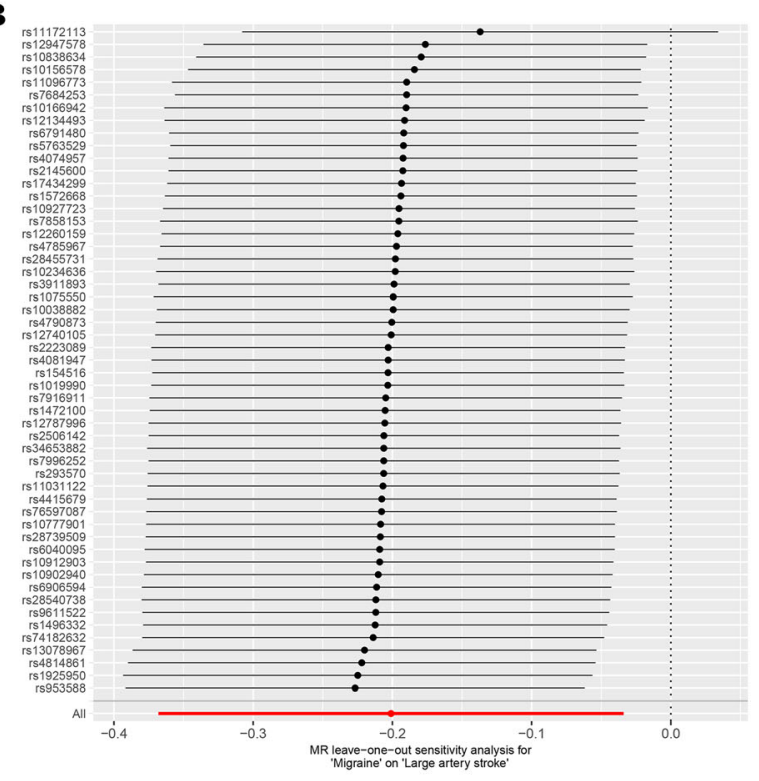

D

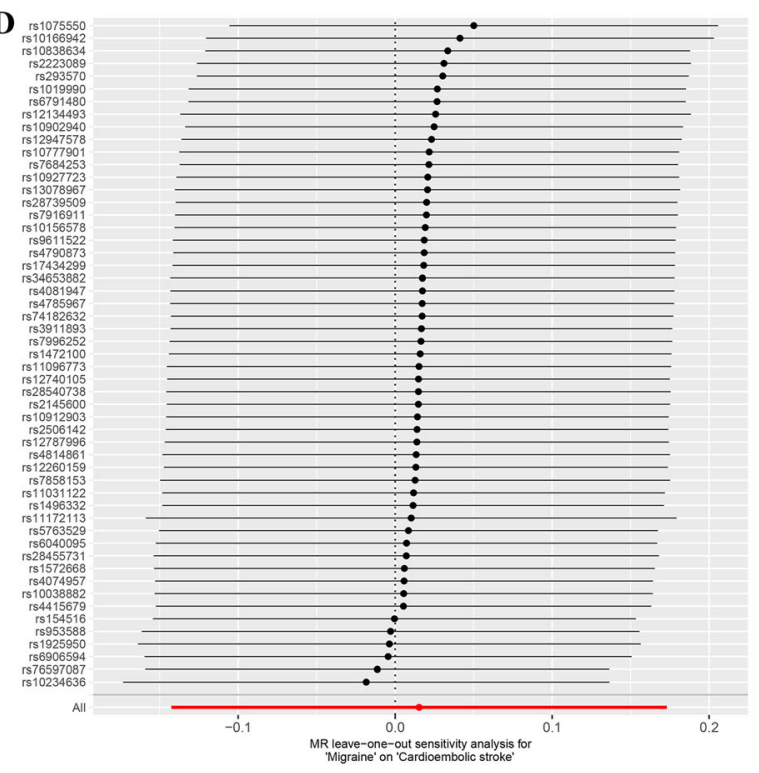

in turn. The red dot and bar indicate the overall estimate and $95 \% \mathrm{CI}$ using the fixed-effect inverse-varianceweighted method

that migraine without aura showed a much stronger overlap with ischemic stroke than migraine with aura, and scores derived from migraine with aura demonstrated a very weak association with ischemic stroke. This does not explain the fact that the association between ischemic stroke and migraine in 
epidemiological studies is most often found in migraine with aura, and not with migraine without aura. Moreover, another recent study disproved the genetic correlation between stroke and migraine when using cross-trait linkage disequilibrium score regression analysis [24]. The study also reported the genetic correlation between migraine and major risk factors for stroke, such as heart disease, type 2 diabetes, blood lipid levels, and blood pressure. For SNPs associated with migraine at a genome-wide significance threshold, we also identified their association with vascular events, BMI, and systolic blood pressure. When we evaluated our results after excluding these pleiotropic SNPs, we determined that migraine has no causal relationship with stroke. This study shows no causal relationship between migraine and ischemic stroke from a genetic perspective, which suggests that migraine is not a modifiable vascular risk factor for ischemic stroke.

Our results also suggest that the observed association between migraine and stroke in epidemiological studies might be susceptible to residual confounders [10]. One possible reason is that most studies, especially prospective studies, collect information on headache status prior to cerebrovascular events. However, any cerebrovascular event can trigger a migrainelike attack, and increased frequency of migraine aura may be a symptomatic manifestation of underlying stroke risk factors (e.g., iron-containing hemosiderin deposition, arterial embolism, cardiogenic embolism) [25]. Therefore, the observed association should not be interpreted simply as a causal relationship between migraine with aura and ischemic stroke.

The strengths of this study include the twosample MR study design, multiple outcomes of stroke and its subtypes, the large sample size, and the use of multiple sensitivity analyses. One weakness of this study is that our data source is from Europe, which may limit the generalizability of our study to populations in other regions. Second, as we applied a two-sample MR study from two different studies, there might be unresolved heterogeneity between studies [26]. In addition, there is some degree of overlap between participants included in the GWAS for migraine and ischemic stroke, which could lead to biased estimates, although the true proportion is likely very small. Finally, it is unlikely that all three key assumptions of MR studies are met in practice, so the biased estimates of causal inference cannot be completely removed [26]. However, MR-Egger regression, MR-PRESSO, and a series of sensitivity analyses in our MR study were performed, and no clear horizontal pleiotropy was found.

\section{CONCLUSIONS}

In conclusion, this study does not support a causal relationship between migraine and ischemic stroke and its subtypes. Our findings suggest that the reported association in previous epidemiological studies might have been confounded by vascular risk factors. Further efforts to investigate the etiology between migraine and ischemic stroke are still warranted.

\section{ACKNOWLEDGEMENTS}

We gratefully acknowledge the Genetic Epidemiology Research on Adult Health and Aging (GERA) and the UK Biobank (UKB) project for providing public available GWAS summarized data for migraine. We gratefully acknowledge the MEGASTROKE project for providing public available GWAS summarized data for ischemic stroke and its subtypes.

Funding. The study was funded by the "13th Five-Year" National Science and Technology Major Project for New Drugs (grant number: 2019ZX09734001), National Natural Science Foundation of China (grant number: 81971138), and the Strategic Priority Research Program "Biological basis of aging and therapeutic strategies" of the Chinese Academy of Sciences (Grant number: XDB39040300). The journal's Rapid Service Fee was funded by the authors.

Authorship. All named authors meet the International Committee of Medical Journal Editors (ICMJE) criteria for authorship for this article, take responsibility for the integrity of 
the work as a whole, and have given their approval for this version to be published.

Authors' Contributions. Mei-Jun Shu, JiaRui Li, and Hang Shen planned the study; MeiJun Shu and Jia-Rui Li contributed to data collection; Mei-Jun Shu and Yi-Cheng Zhu analysed the data; Mei-Jun Shu, Jia-Rui Li, Yi-Cheng Zhu and Hang Shen interpreted the findings and wrote the manuscript; Mei-Jun Shu, YiCheng Zhu and Hang Shen provided revisions of the manuscript. Mei-Jun Shu, Jia-Rui Li, YiCheng Zhu and Hang Shen are responsible for the overall content.

Disclosures. Mei-Jun Shu, Jia-Rui Li, YiCheng Zhu and Hang Shen have nothing to disclose.

Compliance with Ethics Guidelines. This article is based on previously conducted studies and does not contain any new studies with human participants or animals performed by any of the authors.

Data Availability. Data and material are available from corresponding GWAS consortium.

Open Access. This article is licensed under a Creative Commons Attribution-NonCommercial 4.0 International License, which permits any non-commercial use, sharing, adaptation, distribution and reproduction in any medium or format, as long as you give appropriate credit to the original author(s) and the source, provide a link to the Creative Commons licence, and indicate if changes were made. The images or other third party material in this article are included in the article's Creative Commons licence, unless indicated otherwise in a credit line to the material. If material is not included in the article's Creative Commons licence and your intended use is not permitted by statutory regulation or exceeds the permitted use, you will need to obtain permission directly from the copyright holder. To view a copy of this licence, visit http://creativecommons.org/licenses/by$\mathrm{nc} / 4.0 /$.

\section{REFERENCES}

1. Vos T, Abajobir AA, Abate $\mathrm{KH}$, et al. Global, regional, and national incidence, prevalence, and years lived with disability for 328 diseases and injuries for 195 countries, 1990-2016: a systematic analysis for the Global Burden of Disease Study 2016. Lancet (London, England). 2017;390(10100):1211-59.

2. Mahmoud AN, Mentias A, Elgendy AY, et al. Migraine and the risk of cardiovascular and cerebrovascular events: a meta-analysis of 16 cohort studies including 1,152,407 subjects. BMJ Open. 2018;8(3):e020498.

3. Gryglas A, Smigiel R. Migraine and stroke: what's the link? What to do? Curr Neurol Neurosci Rep. 2017;17(3):22.

4. Lantz M, Sieurin J, Sjölander A, Waldenlind E, Sjöstrand C, Wirdefeldt K. Migraine and risk of stroke: a national population-based twin study. Brain. 2017;140(10):2653-62.

5. Tietjen GE. Migraine as a systemic disorder. Neurology. 2007;68(19):1555-6.

6. Eikermann-Haerter K. Spreading depolarization may link migraine and stroke. Headache. 2014;54(7):1146-57.

7. Bochud M (2008) On the use of Mendelian randomization to infer causality in observational epidemiology. Eur Heart J. 2008; 29:2456-57.

8. Choquet H, Yin J, Jacobson AS, et al. New and sexspecific migraine susceptibility loci identified from a multiethnic genome-wide meta-analysis. Commun Biol. 2021;4(1):864.

9. Malik R, Chauhan G, Traylor M, et al. Multiancestry genome-wide association study of 520,000 subjects identifies 32 loci associated with stroke and stroke subtypes. Nat Genet. 2018;50(4):524-37.

10. Lawlor DA, Harbord RM, Sterne JAC, Timpson N, Davey SG. Mendelian randomization: using genes as instruments for making causal inferences in epidemiology. Stat Med. 2008;27(8):1133-63.

11. Turk WE, Uiterwijk A, Pasmans R, Meys V, Ayata C, Koehler PJ. Aspirin prophylaxis for migraine with aura: an observational case series. Eur Neurol. 2017;78: 287-9.

12. Maggioni F, Bruno M, Mainardi F, Lisotto C, Zanchin G. Migraine responsive to warfarin: an update on anticoagulant possible role in migraine prophylaxis. Neurol Sci Off J Ital Neurol Soc Ital Soc Clin Neurophysiol. 2012;33(6):1447-9. 
13. Emdin CA, Khera AV, Kathiresan S. Mendelian Randomization. JAMA. 2017;318(19):1925-6.

14. Gormley P, Anttila V, Winsvold BS, et al. Metaanalysis of 375,000 individuals identifies 38 susceptibility loci for migraine. Nat Genet. 2016;48(8): 856-66.

15. Auton A, Brooks LD, Durbin RM, et al. A global reference for human genetic variation. Nature. 2015;526(7571):68-74.

16. Burgess S, Thompson SG. Avoiding bias from weak instruments in Mendelian randomization studies. Int J Epidemiol. 2011;40(3):755-64.

17. Nikolakopoulou A, Mavridis D, Salanti G. How to interpret meta-analysis models: fixed effect and random effects meta-analyses. Evid Based Ment Health. 2014;17(2):64.

18. Bowden J, Del Greco MF, Minelli C, Davey Smith G, Sheehan N, Thompson J. A framework for the investigation of pleiotropy in two-sample summary data Mendelian randomization. Stat Med. 2017;36(11):1783-802.

19. Hemani G, Zheng J, Elsworth B, et al. The MR-Base platform supports systematic causal inference across the human phenome. Elife. 2018;7:e34408.

20. Verbanck M, Chen C-Y, Neale B, Do R. Detection of widespread horizontal pleiotropy in causal relationships inferred from Mendelian randomization between complex traits and diseases. Nat Genet. 2018;50(5):693-8.

21. Kurth T, Chabriat H, Bousser M-G. Migraine and stroke: a complex association with clinical implications. Lancet Neurol. 2012;11(1):92-100.

22. Etminan M, Takkouche B, Isorna FC, Samii A. Risk of ischaemic stroke in people with migraine: systematic review and meta-analysis of observational studies. BMJ. 2005;330(7482):63.

23. Malik R, Freilinger T, Winsvold BS, et al. Shared genetic basis for migraine and ischemic stroke: a genome-wide analysis of common variants. Neurology. 2015;84(21):2132-45.

24. Siewert KM, Klarin D, Damrauer SM, et al. Crosstrait analyses with migraine reveal widespread pleiotropy and suggest a vascular component to migraine headache. Int J Epidemiol. 2020;49(3): 1022-31.

25. Vongvaivanich K, Lertakyamanee P, Silberstein SD, Dodick DW. Late-life migraine accompaniments: a narrative review. Cephalalgia. 2015;35(10): 894-911.

26. Burgess S, Butterworth AS, Thompson JR, et al. Guidelines for performing Mendelian randomization investigations. Wellcome open Res. 2019;4: 186. 